\title{
Commentary on "Psychophysiological and Cultural Correlates Undermining a Survivalist Interpretation of Near-Death Experiences"
}

\author{
Bruce Greyson, M.D. \\ Department of Psychiatry and Neurobehavioral \\ Sciences, University of Virginia
}

\begin{abstract}
Keith Augustine has provided a legitimate and cogent critique of a transcendental interpretation of near-death experiences, exposing weaknesses in the research methodology, paucity of the data, and gaps in the arguments. He offers evidence from psychophysiological and cultural correlates of NDEs that he interprets as favoring a hallucinatory understanding of these phenomena. However, his analysis relies on idiosyncratic definitions of psychological concepts, reads unidirectional causality into bivariate correlations, and underestimates the empirical predictions of the separation hypothesis. Despite less than compelling evidence for the transcendental hypothesis, it accounts for NDE phenomenology better than the materialist model.
\end{abstract}

KEY WORDS: near-death experiences; dissociation; temporal lobe; brain stimulation; mind-body models.

Keith Augustine sets forth serious challenges to a transcendental or "mind-brain separation" interpretation of near-death experiences (NDEs), and cites evidence from psychophysiological and cultural correlates of NDEs that he interprets as favoring a neurological or

Bruce Greyson, M.D., is the Chester F. Carlson Professor of Psychiatry and Neurobehavioral Sciences and Director of the Division of Perceptual Studies at the University of Virginia. Reprint requests should be addressed to Dr. Greyson at the Division of Perceptual Studies, Department of Psychiatry and Neurobehavioral Sciences, University of Virginia Health System. P. O. Box 800152, Charlottesville, VA 22908-0152; e-mail: cbg4d@virginia.edu. 
sociological understanding of these phenomena. While he raises worthwhile questions, however, his analysis relies on idiosyncratic definitions of psychological concepts, reads unidirectional causality into bivariate correlations, and underestimates the empirical predictions of the separation hypothesis.

Augustine acknowledges that "current psychophysiological models do not fully explain out-of-body experiences (OBEs) and NDEs" and that "psychophysiological correlates ... do not definitively identify their precise causes." But despite these acknowledged weaknesses, he still believes that NDEs "represent internally generated fantasies rather than genuine perceptions of a transcendental environment." I will argue that correlates of NDEs in fact have little to say about their origin and do not imply that they are "internally generated fantasies."

\section{Psychophysiological Correlates}

Augustine starts the section on "Psychophysiological Correlates" by identifying NDEs appropriately as a type of altered state of consciousness (ASC). Then he asserts, inaccurately, that ASCs "are typically understood to involve turning one's attention inward, into the contents of one's own mind, rather than revealing anything about the external world." That misleading characterization of ASCs leads logically to the conclusion that OBEs and NDEs "do not reflect any objective existence outside of the normal physical body." But ASCs do not blind us to the outside world. Whereas some ASCs, such as sleep, do involve turning inward, others demonstrably do not. Indeed, the hyperalertness and intense focus of athletes when they are "in the zone" is a type of ASC, but athletes in that particular altered state have more accurate perceptions of external events than people in the "normal" alert waking state. Arnold Ludwig, who coined the term altered state of consciousness, included in his examples not only states of decreased awareness of the external world but also states of increased awareness (Ludwig, 1966; Ludwig and Lyle, 1964). So the fact that OBEs and NDEs are ASCs, which Augustine correctly defines as "temporary departures from the normal (alert) waking state," does not by any means suggest that they "do not reflect any objective existence outside the normal physical body."

Next Augustine focuses on the correlation between NDEs and the closely-related constructs of dissociation, absorption, and fantasyproneness. Augustine defines dissociation as "the shutting out of 
sensory stimuli" and absorption as "focusing on the imagination." Neither of those definitions comes close to the standard clinical use of these terms. Dissociation is "the telescoping of the attentional field to concentrate on a narrow range of experience and the concomitant exclusion of other material (internal or external) from awareness" (Butler, 2006, p. 45), resulting in the separation of mental processes that are normally experienced as integrated. Dissociation is definitely not "the shutting out of sensory stimuli." When people dissociate, they can still perceive the external world accurately, although those perceptions may be divorced from other aspects of the environment or from the usual emotional reactions. Although most research into dissociation has focused on its role as a defense against trauma, the majority of dissociative experiences are normal and nonpathological (Butler, 2006).

People in pleasant situations often dissociate as a way of enhancing their sensory perceptions, not of withdrawing into fantasy. More than a third of normal volunteers reported dissociating during positive experiences, such as intense athletic competition, sexual encounters, communion with nature, and enjoying music (Pica and Beere, 1995). In such circumstances, attention narrows to a particular meaningful aspect of the external sensory environment. In these dissociations, whose distinction from transcendental experience may be "simply a matter of semantics" (Pica and Beere, 1995, p. 244), sensory perception may be enhanced, and attention is not turned inward toward imagination. Kenneth Ring and Christopher Rosing (1990) and Bruce Greyson (2000) found that near-death experiencers scored higher than a comparison group on a dissociation scale, but their scores were much lower than those of patients with dissociative disorders. This suggests that near-death experiences are adaptive responses to serious stress, rather than pathological (Greyson, 2001).

Related to dissociative tendencies is absorption, the ability to focus one's attention either on selected sensory experiences or on internal imagery to the diminution of other mental activity. Auke Tellegen and Gilbert Atkinson (1974), who coined the term absorption, defined it as "a 'total' attention, involving a full commitment of available perceptual, motoric, imaginative and ideational resources to a unified representation of the attentional object" $(1974$, p. 274 , italics in the original). One can certainly become "absorbed" in imagination, but one also can (and more usually does) become "absorbed" in perception of the external world, such as reading a book, watching a movie, or 
listening to a conversation so intently that one does not perceive other things in the environment. An adaptive advantage of absorption is that it "allows for the full commitment of attention to activity and a reduction in distractibility and self-consciousness and may, therefore, enhance performance in skilled activities (such as sports or performing) or enhance the flow of creativity" (Butler, 2006, p. 56). Thus the moderate association of NDEs with dissociation and absorption does not by any means imply that NDE content is based on imagination.

Related to dissociation and absorption is fantasy proneness, characterized by frequent and vivid fantasies and even hallucinations, intensely vivid sensory experiences, and eidetic imagery (Wilson and Barber, 1981, 1983). In noting the (again, moderate) association between NDEs and a fantasy-proneness, Augustine highlights fantasy-prone individuals" "strong investment in fantasy life," but he glosses over their more intense sensory experiences, leaving the mistaken impression that fantasy-prone individuals mistake fantasy for reality - which was specifically denied by the psychologists who developed the concept (Wilson and Barber, 1983): they stated unequivocally that fantasy-prone people have as good reality-testing as anyone else. However, this is a moot point, because there is absolutely no evidence that NDErs are fantasy-prone individuals. Although NDErs do score higher than nonNDErs on standard measures of fantasy-proneness, which may suggest nothing more than that their sensory perceptions of the outside world are much more vivid than those of nonNDErs, NDErs' scores do not come anywhere near the cut-off point on those measures for designation as a "fantasy-prone personality."

Augustine writes that the "hypothetical status [of dissociation] as a defense mechanism makes much more sense if OBEs and NDEs do not literally involve any form of disembodiment." But if OBEs and NDEs were truly a disembodiment, they would be even more effective in helping a victim escape from a traumatic situation than if they were mere mental illusions. That is, being able to leave one's body during the trauma would be a much more effective defense for a victim than a mere illusion of leaving the body. Thus the defensive value of dissociation argues for rather than against OBEs and NDEs representing real disembodiment.

Augustine goes on to write that the correlation between OBEs and NDEs and "capacity for imaginative involvement" does not make sense if something actually leaves the body. He argues that he would 
not expect psychological crises "as opposed to physiological crises alone" to trigger separation from the body. But psychological threats can be just as terrifying as physiological ones, and it makes perfect sense for a person with an unusually rich "capacity for imaginative involvement" - and therefore greater ability to foresee a potential threat - to flee the body in anticipation of trauma.

Augustine notes the correlation between OBEs and habitually dreaming in a bird's-eye view or seeing oneself during dreams. He interprets this correlation as evidence that OBEs are fantasies conditioned by one's dream life, and writes that physiological models alone predict that people who can induce OBEs would have better dream control skills than spontaneous OBErs. But these correlations are also predicted by a disembodiment model: leaving the body teaches one how to visualize oneself from another perspective, making bird'seye views of oneself during dreams more likely; and repeated induction of OBEs would reinforce these dream control skills far more than occasional spontaneous OBEs. Later he argues that physiological models predict these correlations, whereas the disembodied model must struggle to accommodate them; but that conclusion reflects his enchantment with the materialist model. Someone with a different perspective might as honestly say that physiological models must struggle to accommodate these correlations that are predicted by the disembodiment model.

Still later Augustine states that Susan Blackmore's theory of OBEs as perceptual distortions (Blackmore, 1993) is supported by its predictions that OBErs have better imagery skills and visuospatial skills. But the separation hypothesis also predicts that OBErs, because of the visuospatial training they receive in their OBEs, should have better imagery and visuospatial skills.

Throughout this discussion, Augustine consistently interprets correlations between OBEs and psychological traits as implying a unidirectional causal effect of the psychological variables on OBEs. But it is at least as plausible that OBEs, particularly repeated or vivid ones, may lead to these psychological changes. One cannot assume causation from correlation. Almost any human experience is likely to be correlated with a host of psychological and physiological factors, because we are whole beings with integrated psychology and physiology. Certainly an experience as profound as leaving the body would have psychological effects that would manifest as correlates to psychological traits; and it is plausible that certain psychological traits 
might make it easier for someone to leave the body under stress. There is no theoretical reason to link psychological correlates to a psychophysiological model in preference to a separation model.

Augustine next turns to correlations between physiological conditions of a close brush with death and features of the NDE, again drawing sweeping conclusions from rather small correlations. It is true that some features are relatively more common in NDEs from various causes, but there is no feature of NDEs in one physiological condition, such as cardiac arrest, traumatic accident, drug overdoses, respiratory arrests during surgery, and so on, that is not seen in all the others. I see no reason why these relative frequencies should count as evidence that NDEs are hallucinations.

Augustine writes that "if NDEs occur when consciousness is released from the confines of the brain, then altering brain chemistry ought not to have an effect on NDE content." But all we know about NDE content is what experiencers choose to tell us about what they are able to put into words of what they can recall about their NDE content. Regardless of whether or not altered brain chemistry affects NDE content, it certainly affects one's ability to recall, understand, and verbalize, so it is not at all surprising that experiencers with altered brain chemistry report different things than experiencers without altered brain chemistry (or with differently altered brain chemistry). In other words, reports of NDEs are necessarily filtered through the brain and linguistic patterns of the experiencer, whether the actual NDE occurred when consciousness was in or out of the brain. Because altered brain chemistry affects memory and linguistic facility, its influence on NDE reports tells us nothing about the cause of the NDE itself.

\section{Is the Temporal Lobe Implicated in OBEs?}

Augustine writes that "a preponderance of the evidence ... implicates the temporal lobe in the production of NDEs," including electrical stimulation studies of the temporal lobe and observations of patients with temporal lobe seizures. In fact, most of that evidence does not involve coherent NDE-like experiences but rather fragmentary bits of music or singing, seeing isolated and repetitive scenes that seemed familiar, hearing voices, experiencing fear or other negative emotions, or seeing bizarre imagery that was often described as dream-like, distorted experiences quite unlike NDEs (Gloor, 1990; 
Gloor, Olivier, Quesney, Andermann, and Horowitz, 1982; Penfield, 1955; Penfield and Perot, 1963, pp. 611-665). Neuropsychiatrist Peter Fenwick concluded that "abnormal discharges in the temporal lobe may produce confusional fragments of phenomena sometimes seen in NDEs. ... This is a very long way from arguing that seizure discharges in those areas, resulting from brain catastrophe, can give rise to the clearly remembered, highly structured NDE (Fenwick, 1997, p. 48).

Electrical stimulation studies have produced not OBEs but rather trivial optical illusions whose experiencers do not believe themselves to have left their bodies. Despite these experiences being labeled by some researchers as OBEs, they are not. For example, in a recent report of the purported induction an out-of-body experience by brain stimulation, the single subject reported only a sensation as of "her body falling/drifting side wards and even out of the chair" (Schutter, Kammers, Enter, and Van Honk, 2006, p. 240). This illusion of the body moving has no relevance to out-of-body experiences, despite the authors' claims to have induced an OBE. Augustine, to his credit, acknowledges that these electrically stimulated illusions are "clearly distinct from" spontaneous OBEs. Certainly they are not experienced as the same: subjects in electrical stimulation experiments describe what they thought was an illusion that felt as if they were out of their bodies (or, more commonly, "half in and half out"), but they did not believe they actually were; whereas NDErs describe what they thought was a real experience of actually being separate from the physical body.

Electrical stimulation at most produces only a sense of perception of things visible from the physical position of the individual's eyes, and those perceptions disappear when the eyes are closed or the person loses consciousness. Electrical brain stimulation has never produced accurate perception of anything not visible to the physical eyes, or that persists when the eyes are closed, or that is from an out-of-body perspective - all typical features of spontaneous OBEs (GieslerPetersen, in press; Holden, Long, and MacLurg, 2006).

Despite Augustine's acknowledgment that the electrically-induced illusion "lacks the realism, continuity, and stability characteristic of spontaneous OBEs," he is not ready to concede that they are different phenomena. He offers as one hypothesis that electrically induced bodily illusions and spontaneous OBEs may be variants or "different parts of a single complex neural mechanism of generating OBEs." This is in principle a plausible hypothesis, but without specifying what that 
"single complex neural mechanism" might be is it an unfalsifiable hypothesis, and therefore of no scientific interest. We may someday uncover evidence suggesting that electrically induced bodily illusions and spontaneous OBEs are variants of the same phenomenon, but at present there is no evidence to suggest that these vastly different experiences are any more alike than, for example, reading a news report about war and actually participating in combat. Both experiences might provoke common emotional reactions and activate common brain sites, but they are clearly not variants of the same experience or "parts of a single complex neural mechanism." Though we might use some of the same terms to describe reading about war and participating in combat, they would differ markedly in the attribution of "reality" to the experience and in their (particularly long-term) after-effects.

Augustine notes that it is not unheard of for patients to report some features of NDE during their seizures. However, the vast majority of patients with seizures do not experience any of these features. In fact, most patients with temporal lobe epilepsy also have no memory afterward for what happened during a seizure (Fenwick, 1997). Epilepsy specialist Ernst Rodin, who favors a neurological explanation of NDEs, acknowledged bluntly: "In spite of having seen hundreds of patients with temporal lobe seizures during three decades of professional life, I have never come across that symptomatology [of NDEs] as part of a seizure" (1989, p. 256). In the prospective study that Augustine cited (Devinsky, Feldmann, Burrowes, and Bromfield, 1989), only 6 percent of seizure patients described any body image anomalies, let alone OBEs. Furthermore, although these patients had suffered numerous seizures, often over a period of many years, the majority who described body image anomalies reported only one such experience. These findings suggest that localized abnormal activity in the brain is not only not necessary, but also not in general sufficient to produce an OBE (Kelly, Greyson, and Kelly, 2006).

Even if the temporal lobe were shown to be implicated in NDEs (which is far from established), it is not plausible that that part of the brain is producing them. This is because both seizure activity and direct electrical stimulation typically disrupt whatever patterns of neuroelectric activity would otherwise be going on in that part of the brain. Electrical stimulation and seizures are not like physiological electrical activity and do not result simply in a localized "activation" of the stimulated region. As Wilder Penfield (1975), the neurosurgeon 
who first mapped brain regions by stimulating the brain, clearly recognized, the predominant effects of electrical stimulation are disruption of electrical activity in the immediate vicinity of the electrode, accompanied by abnormal patterns of discharge into additional brain areas to which the stimulated cortex itself is linked. The net result of electrical stimulation and with epileptic seizures is a poorly controlled, poorly characterized, and spatially widespread pattern of abnormal electrical activity.

In other words, seizure activity and direct electrical stimulation might explain the failure of normal perceptual integration, but not the production of coherent experiences such as NDEs (Kelly, Greyson, and Kelly, 2006). Electrical stimulation or abnormal electrical discharges in the brain cannot account for complex perception or thought because those disruptions in brain functioning would ordinarily abolish consciousness. This much was acknowledged by the very neurosurgery team Augustine cited, who called consciousness during seizures a "paradox" and "an unresolved problem" (Devinsky, Feldmann, Burrowes, and Bromfield, 1989, pp. 1087-1088).

A more problematic distinction between electrically induced bodily illusions and spontaneous OBEs that challenges the claim that they comparable is that some OBEs include corroborated reports of perception of events at a distance. In many of these cases the events in question included unlikely and unexpected details that had been verified as having occurred, and the experience had been reported to someone else before that verification occurred. The evidence for accurate OBE perception has already been discussed at length in these pages by Augustine (2007a) and the commentaries that followed his article, and need not be repeated here. Suffice it to say that the evidence is not strong enough to compel belief in veridical OBE perception, yet it is too strong to be swept under the rug as "misperception" without any evidence for that unfalsifiable hypothesis.

Augustine protested in his previous article that "very few cases of 'veridical perception' during NDEs have been corroborated" (2007a, p. 204). But when Janice Holden (2007) pointed out that Augustine was focusing on only the rare NDEs that contained apparent hallucinatory features, he countered that the presence of even one hallucinatory feature would be enough to prove his point: "What is problematic for a survivalist interpretation is not the frequency of such NDEs, but that they occur at all" (2007b, p. 59, italics in the original). By that same line of reasoning, what is problematic for a hallucinatory 
interpretation of NDEs is not the frequency of veridical out-of-body perception, but that they occur at all. In fact, Holden (2007) found that veridical $O B E$ perception was not rare: more than a third of the published cases of accurate OBE perception in NDEs were corroborated by independent, objective sources.

In discussing the EEG study of NDErs by Willoughby Britton and Richard Bootzin (2004), Augustine notes that NDErs were four times as likely to have "temporal lobe epileptiform EEG activity" during sleep as were nonNDErs. What Britton and Bootzin actually reported was that "No clinically significant seizure activity (EEG or tonic-clonic posturing) was observed in either group" (Britton and Bootzin, 2004, p. 255). They did find indirect suggestion of subclinical temporal lobe activity that was not suggestive of seizures in 22 percent of NDErs and only 5 percent of nonNDErs. If this subclinical activity in the temporal lobe, which has absolutely no clinical significance, was involved in producing NDEs, why was it absent in three-fourths of the NDErs? The NDErs in this study had better positive coping skills than the nonNDErs, but the groups did not differ on maladaptive stress responses, suggesting that whatever differentiated the NDErs from the nonNDErs was an enhanced function rather than a dysfunction.

Augustine also notes that NDErs as a group scored higher on a measure of "epileptic signs typical of temporal lobe activity," but the concept of whether there are "epileptic signs typical of temporal lobe activity" remains controversial after centuries of conflicting anecdotal observations (Shetty and Trimble, 1997). Studies of personality traits of epileptic patients have been plagued by methodological problems associated with diagnostic uncertainties and difficulties operationalizing some of the purported psychophysiological correlates of temporal lobe epilepsy. It appears likely that patients who have right temporal lobe seizures do differ from others in certain ways, and a list of the character traits included in standard measures of a "temporal lobe personality" includes many features common among near-death experiencers. Temporal lobe epileptics and NDErs, for example, are both reported to have deeper emotions, elation, increased moral sensitivity, a sense of personal destiny, desire to write detailed notes and even books, deep religious beliefs, and interest in philosophical issues.

But that common association does not necessarily mean that NDErs are happy, interested in spirituality and philosophy, feel a sense of destiny and want to write about it because their right temporal lobes 
have been damaged. There are other, more obvious reasons for a person who has had a profound mystical experience to feel deep emotions, elation, and deep religious and philosophical beliefs. As an analogy, if one stays up all night, one is likely to be tired and confused the next day, with difficulty thinking and speaking clearly. The fact that those symptoms may also be associated with alcohol intoxication does not make us suspect the sleep-deprived person of having imbibed; the sleep deprivation itself is sufficient explanation. The fact that temporal lobe dysfunction can mimic (a few of) the psychospiritual effects of NDEs does not imply that the experiencer has damaged temporal lobes; the experience itself is sufficient explanation. There is a tautological character to the argument that defines certain NDE features and aftereffects as epileptic signs (or hallucinations or dissociative symptoms) and then presents the same NDE features and aftereffects as evidence that experiencers are unreliable witnesses because they have epilepsy (or hallucinate or dissociate).

\section{Cultural Differences}

Prior beliefs do influence reports of near-death experiences. The life review and tunnel sensation, for example, are common in some cultures but rare in others (Kellehear, 1993). Such cultural differences lend support to the view that the specific content of NDEs can be colored by the sociocultural context in which they occur.

Other data, however, do not support the expectation theory. People who had no prior knowledge about NDEs have described the same kinds of experiences and features as have people who were more familiar with the phenomenon (Greyson, 1991; Greyson and Stevenson, 1980; Ring, 1980; Sabom, 1982). And as Augustine notes, there is ample evidence from a variety of sources that experiences that were reported before 1975, when Raymond Moody's first book coined the term NDE and made it a well-known phenomenon, do not differ from those that occurred after that date.

Augustine suggests that when NDE imagery contradicts personal and cultural beliefs, they can be explained as being influenced by subconscious expectations of which the experiencer is unaware. As a clinical psychiatrist, I am well aware of the influence of subconscious processes on perception. However, subconscious expectations are not "Get Out of Jail Free" cards that can be invoked at any time to avoid facing inconvenient facts. If the existence of a subconscious expecta- 
tion is not supported by independent evidence, then it has no more explanatory power than any other unfalsifiable hypothesis, such as divine intervention or fraud.

Augustine does raise legitimate questions about the role of prior beliefs on NDEs of children and of nonWestern experiencers, questions whose importance researchers have underestimated. But raising these questions is not tantamount to answering them. Augustine's cogent critique of the evidence demonstrates that "prototypical" Western NDE features are not universal across cultures; but it does not demonstrate that NDE features are determined by expectation.

The crosscultural differences observed are consistent with the hypothesis that expectation influences the core experience, but also with the hypothesis that expectation influences how people interpret what they have experienced. This is not a new argument: two decades ago researchers recognized that any underlying core experience, if indeed there is one, is "inevitably cast in the images, concepts and symbols available to the individual" (Roberts and Owen, 1988, p. 611; see also Knoblauch, Schmied, and Schnettler, 2001).

Augustine argues that we should take experiencers' descriptions as literal descriptions of what they encountered and not consider the effect of belief on interpretation of experiences: "The most straightforward reason why different NDErs would describe their experiences in different ways is because they actually have very different experiences. ... [D]ifferent people would naturally be expected to report similar experiences if they were traveling to the same afterlife environment" (italics in the original).

I do not share Augustine's expectation that people with different backgrounds and interests would "naturally" report the same things. The varying terminology and crosscultural differences in NDE accounts may argue against a physiological explanation for NDEs, but they do not discredit the reality of the experiences. Individual differences in cultural expectations influence our perceptions of the physical world; why should we expect them not to influence our perceptions of a transcendental dimension, if one exists - particularly when the NDErs report, as many do, that what they experienced was ineffable?

As an analogy, imagine that three of your acquaintances describe to you their alleged visit to a place they call "France." One, a hedonistic gourmand, details the sumptuous meals she enjoyed, but does not mention architecture or people. The second, a religious artist, 
describes in detail the magnificent cathedrals with their paintings and stained glass windows, but does not mention food or people. The third, a high-powered businessman, rails about the rude taxi drivers and street merchants, but does not mention food or architecture. Their descriptions of their purported visits to a place called "France" have little in common; furthermore, their "perceptions" of "France" largely conform to their expectations and their interests. Do you assume therefore that their visits to "France" took place only in their imaginations, and that no such realm actually exists?

I agree with Augustine that we are not justified in assuming that diverse NDE descriptions are different ways of describing the same locale, just as we have no reason to assume that these three travelers actually visited the same country they call "France." But we are also not justified in using the discrepancies as evidence that the NDE world does not exist, any more than we can use our travelers' varied accounts as evidence that "France" does not exist. The variations in description are evidence of differences in perception, but do not by themselves allow us to make judgments about what was perceived.

\section{The Real Challenge}

Although we may eventually find that physiological, psychological, and sociocultural factors interact in complicated ways in conjunction with NDEs, theories proposed thus far consist largely of unsupported speculations about what might be happening during an NDE. None of the proposed neurophysiological mechanisms have been shown to occur in NDEs.

The most important objection to current psychophysiological theories is that mental clarity, vivid sensory imagery, a clear memory of the experience, and a conviction that the experience seemed more real than ordinary consciousness are the norm for NDEs, even when they occur under conditions of drastically altered cerebral physiology. As Sam Parnia and Peter Fenwick pointed out, "any acute alteration in cerebral physiology such as occurring in hypoxia, hypercarbia, metabolic, and drug induced disturbances and seizures leads to disorganised and compromised cerebral function ... [and] impaired attention," whereas "NDEs in cardiac arrest are clearly not confusional and in fact indicate heightened awareness, attention and consciousness at a time when consciousness and memory formation would not be expected to occur" (2002, p. 8). Moreover, experiencers of 
NDEs in connection with cardiac arrest almost invariably retain vivid memories of their experience that change little with the passage of time (van Lommel, van Wees, Meyers, and Elfferich, 2001), despite the fact that memory under such conditions is ordinarily seriously impaired.

The challenge for explanatory models of NDEs is to take into account the vivid and complex thinking, sensations, and memory formation under conditions in which current neuroscientific models of the mind deem them impossible, such as under general anesthesia and in cardiac arrest (Kelly, Greyson, and Kelly, 2006). This continuation and even enhancement of mental functioning at a time when the brain is physiologically impaired present problems for the mind-brain identity model. Only when researchers approach the study of NDEs with this paradox firmly in mind will we progress in our understanding of NDEs beyond tenuous and untestable neuroscientific conjectures.

Augustine argues that a scientific explanation of NDEs must be considered in the context of a scientific explanation of all human experience. He believes that we have good evidence for hallucinations, but that we do not have such evidence for the separation of mind from brain, and that therefore it would be more parsimonious to view NDEs as hallucinations than as instances of mind-brain separation.

I agree that NDEs should be evaluated in a larger context of human experiences rather than as isolated events. But there is in fact an enormous body of scientific evidence for mind-brain separation from other phenomena, encompassing data related to placebo responses, stigmata, hypnotic suggestion, memory, automatisms, multiple personalities, creative genius, mystical experience, and acquisition of information unavailable to the physical senses (Kelly, Kelly, Crabtree, Gauld, Grosso, and Greyson, 2006; Radin, 1997). In the context of this wealth of other evidence, it becomes quite parsimonious to consider NDEs as one more example of separation of mind from brain.

Without exception, every report of a large study of NDEs published in a mainstream medical journal has concluded that these phenomena cannot be explained as hallucinations. Such unanimity among scientific researchers is unusual and should tell us something. Why is it that scientists who have done the most near-death research believe the mind is not exclusively housed in the brain, whereas those who regard NDEs as hallucinations by and large have not conducted any studies of the phenomenon at all? 
Augustine attributes this consensus to investigator bias: that neardeath research has been influenced by the researchers' beliefs. But in fact he has it backwards: the researchers' beliefs have been influenced by their consistent research findings. Most near-death researchers did not go into their investigations with a belief in mind-body separation, but came to that hypothesis based on what their research found. Indeed, Michael Sabom wrote about entering the field specifically to debunk NDE reports:

When I began my study of the NDE, I was convinced that the NDE would readily be accounted for using some traditional scientific explanation. I have searched for such an explanation over the past five years and have not yet found one that is adequate. In recent years I have begin to consider another approach toward explaining the NDE, and approach which holds open the possibility that the perception of an "out-of-body" experience at the point of death may be accurate, i.e., that it somehow does occur "out-of-body." (1981, p. 46)

\section{Conclusion}

Augustine raises important questions about NDErs' expectations and personalities influencing their experiences, and about researchers' oversimplification of the concept of cultural afterlife beliefs. We would be well advised to attend to these criticisms, to review our methodology, and to seek collaboration with researchers who hold conflicting perspectives. In sum, Augustine has provided a legitimate and cogent critique of near-death research, exposing weaknesses in our methodology, paucity of our data, and gaps in our arguments. For that I thank him. But the hallucinatory model he favors is supported by even fewer data than the separation model, and by speculation and innuendo rather than evidence-based argument.

Augustine claims that the hallucination model of NDEs is superior because it has greater predictive power than a dualistic model. But in fact a dualistic model predicts all the psychophysiological correlates that the hallucination model does, plus the accurate perceptions that are inexplicable if NDEs are just hallucinations. The major advantage of the hallucination model is its compatibility with the materialistic worldview favored by a majority of neuroscientists (though not by a majority of physicists). The major disadvantage of the hallucination model is that it fails to account for the phenomenon, and is plausible 
only if we discredit or discount much of our data. As astronomer and spectroscopy pioneer Paul Merrill quipped, "If you eliminate the data that do not agree, the remaining data agree very well." But disregarding disagreeable data or dismissing them outright as fraud or misperception is the hallmark of pseudoscience, not science. Materialists often claim credit for the scientific advances of the past few centuries. But it is the scientific method of empirical hypothesis testing, rather than a materialistic philosophy, that has been responsible for the success of science in explaining the world. If it comes to a choice between the empirical method and a materialistic worldview, the true scientist will choose the former.

Physiological models of NDEs rely on what Karl Popper (Popper and Eccles, 1977) called "promissory materialism," the position that, although we do not know enough now to explain everything in terms of materialistic models, we will some day. But although promissory materialism is a legitimate philosophical position, it is empirically unfalsifiable, and therefore not a scientific hypothesis. As neurophysiologist John Eccles, who won a Nobel Prize for his work on the synapse, summarized it:

According to promissory materialism ... scientific advance will progressively restrict the phenomena that appear to require mental terms for their explanation, so that in the fullness of time everything will be describable in the materialist terms of the neurosciences. I regard this theory as being without foundation. The more we discover scientifically about the brain the more clearly do we distinguish between the brain events and the mental phenomena and the more wonderful do the mental phenomena become. Promissory materialism is simply a superstition held by dogmatic materialists. It has all the features of a Messianic prophecy, with the promise of a future freed of all problems - a kind of Nirvana for our unfortunate successors. (Eccles, 1994, p. 7)

Indeed, the materialistic lens provides so rosy a view that its proponents often overlook the perspectives offered by other lenses. Augustine presents the hallucinatory model of NDEs in opposition to a transcendental one, as if either one or the other must be true. But NDE phenomenology suggests to me that some NDE features may well be linked to physiological events, some to sociopsychological belief, and others to no known materialist cause. Indeed, many NDErs speak of having "one foot in each world," of being aware simultaneously of the physical environment (including their bodies) and also of a transcendental dimension. Is our thinking so impoverished that 
we cannot imagine an experiencer dissociating awareness of the physical body out of fear, then communicating with nonmaterial entities, and then feeling pulled back into the body by feeling the surgeon's hands massaging the heart? Why must we link all NDE features to one kind of explanation? Augustine argues that parsimony requires us to choose just one model rather than two or three to explain NDEs. But a model is not preferable if it achieves parsimony only by ignoring what it cannot explain.

Augustine is correct that proponents of a transcendental model of NDEs have not provided sufficient data to prove their case. But unless the internally-generated hallucination model starts to address the discomforting data from NDEs rather than ignoring them, it cannot be considered a serious alternative.

\section{References}

Augustine, K. (2007a). Does paranormal perception occur in near-death experiences? Journal of Near-Death Studies, 25, 203-244.

Augustine, K. (2007b). "Near-death experiences with hallucinatory features" defended. Journal of Near-Death Studies, 26, 55-65.

Blackmore, S. (1993). Dying to live: Near-death experiences. Buffalo, NY: Prometheus.

Butler, L. D. (2006). Normative dissociation. Psychiatric Clinics of North America, 29, $45-62$.

Devinsky, O., Feldmann, E., Burrowes, K., and Bromfield, E. (1989). Autoscopic phenomena with seizures. Archives of Neurology, 46, 1080-1088.

Eccles, J. C. (1994). How the self controls its brain. Berlin, Germany: Springer-Verlag.

Fenwick, P. (1997). Is the near-death experience only $N$-methyl- $D$-aspartate blocking? Journal of Near-Death Studies, 16, 43-53.

Giesler-Petersen, I. (In press). Further commentary on "induced OBEs" [Letter]. Journal of Near-Death Studies, 26.

Gloor, P. (1990). Experiential phenomena of temporal lobe epilepsy. Brain, 113, 1673-1694.

Gloor, P., Olivier, A., Quesney, L. F., Andermann, F., and Horowitz, S. (1982). The role of the limbic system in experiential phenomena of temporal lobe epilepsy. Annals of Neurology, 12, 129-144.

Greyson, B. (1991). Near-death experiences precipitated by suicide attempt: Lack of influence of psychopathology, religion, and expectations. Journal of Near-Death Studies, 9, 183-188.

Greyson, B. (2000). Dissociation in people who have near-death experiences: Out of their bodies or out of their minds? Lancet, $355,460-463$.

Greyson, B. (2001). Posttraumatic stress symptoms following near-death experiences. American Journal of Orthopsychiatry, 71, 358-373.

Greyson, B., and Stevenson, I. (1980). The phenomenology of near-death experiences. American Journal of Psychiatry, 137, 1193-1196.

Holden, J. M. (2007). More things in heaven and earth: A response to "Near-death experiences with hallucinatory features." Journal of Near-Death Studies, 26, 33-42.

Holden, J. M., Long, J., and MacLurg, J. (2006). Out-of-body experiences: All in the brain? Journal of Near-Death Studies, 25, 99-107. 
Kellehear, A. (1993). Culture, biology, and the near-death experience: A reappraisal. Journal of Nervous and Mental Disease, 181, 148-156.

Kelly, E. F., Kelly, E. W., Crabtree, A., Gauld, A., Grosso, M., and Greyson, B. (eds.). (2006). Irreducible mind: Toward a psychology for the $21^{\text {st }}$ century (pp. 367-421). Lanham, MD: Rowman and Littlefield.

Kelly, E. W., Greyson, B., and Kelly, E. F. (2006). Unusual experiences near death and related phenomena. In E. F. Kelly, E. W. Kelly, A. Crabtree, A. Gauld, M. Grosso, and B. Greyson (eds.), Irreducible mind: Toward a psychology for the $21^{\text {st }}$ century (pp. 367-421). Lanham, MD: Rowman and Littlefield.

Knoblauch, H., Schmied, I., and Schnettler, B. (2001). Different kinds of near-death experiences: A report on a survey of near-death experiences in Germany. Journal of Near-Death Studies, 20, 15-29.

Ludwig, A. M. (1966). Altered states of consciousness. Archives of General Psychiatry, $15,225-234$.

Ludwig, A. M., and Lyle, W. H. (1964). Tension induction and the hyper-alert trance. Journal of Abnormal and Social Psychology, 69, 70-76.

Moody, R. A. (1975). Life after life. Covington, GA: Mockingbird Books.

Parnia, S., and Fenwick, P. (2002). Near death experiences in cardiac arrest: Visions of a dying brain or visions of a new science of consciousness. Resuscitation, 52, 5-11.

Penfield, W. (1955). The role of the temporal cortex in certain psychical phenomena. Journal of Mental Science, 101, 451-465.

Penfield, W. (1975). The mystery of the mind: A critical study of consciousness and the human brain. Princeton, NJ: Princeton University Press.

Penfield, W., and Perot, P. (1963). The brain's record of auditory and visual experience: A final discussion and summary. Brain, 86, 595-696.

Pica, M., and Beere, D. (1995). Dissociation during positive situations. Dissociation, 8 , 241-246.

Popper, K. R., and Eccles, J. C. (1977). The self and its brain. Berlin, Germany: Springer.

Radin, D. (1997). The conscious universe: The scientific truth of psychic phenomena. San Francisco, CA: HarperEdge.

Ring, K. (1980). Life at death: A scientific investigation of the near-death experience. New York: Coward, McCann and Geoghegan.

Ring, K., and Rosing, C. J. (1990). The Omega Project: An empirical study of the NDEprone personality. Journal of Near-Death Studies, 8, 211-239.

Roberts, G., and Owen, J. (1988). The near-death experience. British Journal of Psychiatry, 153, 607-617.

Rodin, E. (1989). Comments on "A neurobiological model for near-death experiences." Journal of Near-Death Studies, 7, 255-259.

Sabom, M. (1981). The near-death experience: Myth or reality? A methodological approach. Anabiosis: The Journal of Near-Death Studies, 1, 44-56.

Sabom, M. (1982). Recollections of death: A medical investigation. New York: Harper and Row.

Schutter, D. J. L. G., Kammers, M. P. M., Enter, D., and Van Honk, J. (2006). A case of illusory own-body perceptions after transcranial magnetic simulation of the cerebellum. Cerebellum, 5, 238-240.

Shetty, T., and Trimble, M. (1997). The Bear Fedio Inventory: Twenty years on. Journal of Epilepsy, 10, 254-262.

Tellegen, A., and Atkinson, G. (1974). Openness to absorbing and self-altering experiences ("absorption"), a trait related to hypnotic susceptibility. Journal of Abnormal Psychology, 83, 268-277.

van Lommel, P., van Wees, R., Meyers, V., and Elfferich, I. (2001). Near-death experiences in survivors of cardiac arrest: A prospective study in the Netherlands. Lancet, 358, 2039-2045. 
Wilson, S. C., and Barber, T. X. (1981). Vivid fantasy and hallucinatory abilities in the life histories of excellent hypnotic subjects ("somnambules"): Preliminary report with female subjects. In E. Klinger (ed.), Imagery. Vol. 2: Concepts, results, and applications (pp. 133-149). New York: Plenum.

Wilson, S. C., and Barber, T. X. (1983). The fantasy-prone personality: Implications for understanding imagery, hypnosis, and parapsychological phenomena. In A. A. Sheikh (ed.), Imagery: Current theory, research, and application (pp. 340-390). New York: Wiley. 